\title{
A Platform for Alkene Carbofunctionalization with Diverse Nucleophiles
}

\author{
Travis L. Buchanan, Samuel N. Gockel, Alexander M. Veatch, Ya-Nong Wang, Kami. L. Hull* \\ Department of Chemistry, University of Texas at Austin, 100 East $24^{\text {th }}$ Street, Austin, Texas 78712 , United States \\ Supporting Information Placeholder
}

\begin{abstract}
A general system achieving three-component intermolecular carbofunctionalization of alkenes is presented. A range of substituted alkenes are functionalized with $\alpha$-bromo carbonyl electrophiles and nitrogen, oxygen, and carbon nucleophiles. Mechanistic findings support the intermediacy of a cyclic oxocarbenium ion.
\end{abstract}

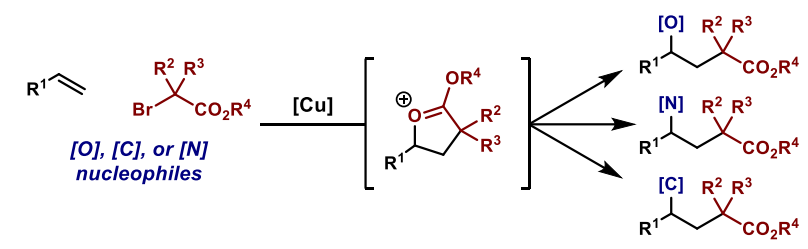

\section{- INTRODUCTION}

Alkene carboheterofunctionalization is a powerful strategy for the construction of small molecules, transforming simple substrates into densely functionalized compounds in a single synthetic operation. The multicomponent nature of transformations of this class enables a high degree of reaction modularity, facilitating access to diverse molecular structures and small-molecule libraries. Significant progress has been made in the development of one- and twocomponent alkene carboheterofunctionalization reactions, such as carboamination $^{1}$ and carboetherification ${ }^{2}$, however the intramolecular nature of these systems restricts modularity and overall versatility.

Despite the value of general, three-component alkene carboamination and carboetherification reactions, few among such methods possess broad scope and wide applicability. This is largely due to chemoselectivity challenges inherent to three-component transformations, wherein bimolecular cross-coupling of any two components with exclusion of the third is often kinetically competitive with the desired difunctionalization (Figure 1). Despite this, significant progress has been made, often within restricted substrate classes, in the areas of three-component alkene carboamination ${ }^{3}$ and carboetherification. ${ }^{4}$

Figure 1. Chemoselectivity Challenges in Multicomponent Alkene Difunctionalization.

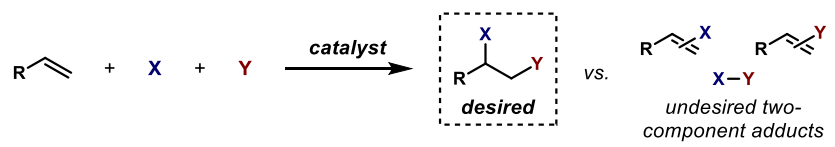

Alkene carboheterofunctionalization strategies employing alkyl radicals have recently flourished, fueled by advances in the mild generation of the requisite open-shell intermediates. This strategy directly addresses chemoselectivity challenges, as the rapid rates with which organoradicals add to olefins ensures that these two

components combine with fidelity. ${ }^{5}$ The resulting adduct of the olefin and organoradical, itself a radical intermediate, is then poised to undergo heterofunctionalization through one of several possible mechanisms. Regiocontrol in such systems is achieved by the inherent tendency of radical species to add to the terminus of $\pi$-systems. The success of this approach is manifest in a recent deluge of reports that utilize these principles to achieve desirable three-component reactivity. ${ }^{6}$

We, independently with the $\mathrm{Li}$ group, ${ }^{7}$ recently developed a method detailing a fully intermolecular three-component alkene carboamination reaction utilizing copper catalysis (Figure 2A). Electronically and sterically diverse vinylarenes and unactivated aliphatic alkenes are combined with readily available $\alpha$-bromo carbonyl electrophiles and amine nucleophiles to afford valuable $\gamma$ aminocarbonyl and iminolactone structures in a single step. Such functionalities appear broadly as key pharmacophores in a wide variety of biologically active molecules such as GABA analogues, opioid analgesics, anti-cancer and anti-inflammatory agents (Figure 2B). ${ }^{8}$

Figure 2. A: Access to diverse $\gamma$-aminocarbonyl compounds through alkene carboamination. B: Prevalence of $\gamma$-aminocarbonyl compounds among biologically active molecules.

A. Previously reported three-component alkene carboamination

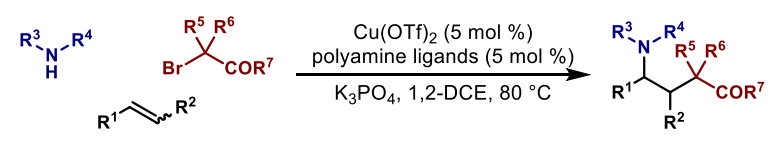

B. Biologically active $\gamma$-aminocarbonyls and $\gamma$-lactams

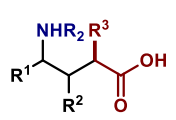

GABA and analogs:

- Methadone $\cdot$ Nomethadone $\cdot$ Atrolate Loperamide $\cdot$ Bezitramide $\cdot$ Tubulsin A

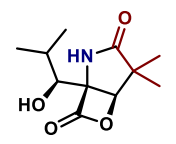

(-)-Methomuralide proteosome inhibitor

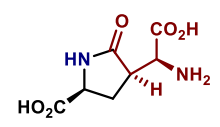

Penmacric Acid anti-inflammatory 
Table 1. Summarized scope of alkene carboamination ${ }^{a}$

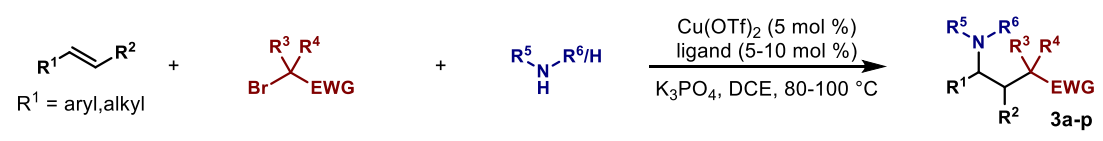

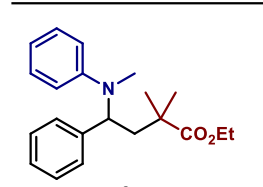

$3 a$
$87 \%$

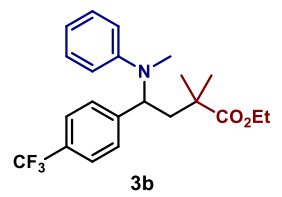

$55 \%$<smiles>CCOC(=O)C(C)(C)CC(c1ccc(OC)cc1)n1c2ccccc2c2ccccc21</smiles>

$3 \mathbf{g}$
$76 \%$

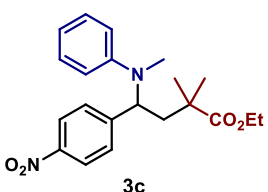

$54 \%$

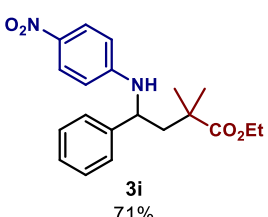

$71 \%$

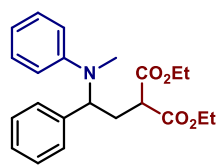

$3 n$
$61 \%$

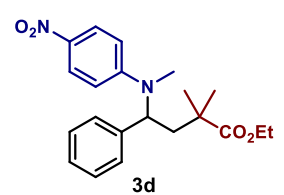

$60 \%$

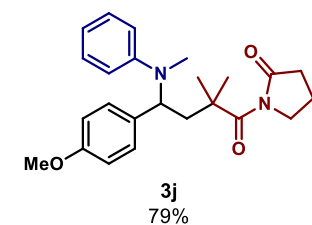

$3 \mathbf{j}$
$79 \%$

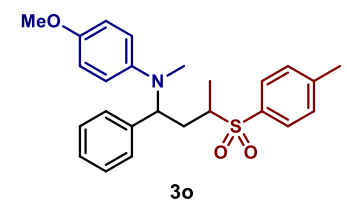

$67 \%, 10: 1$ d.r.

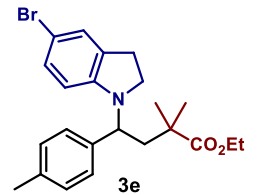

$70 \%$

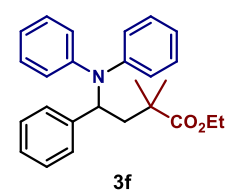

$68 \%$

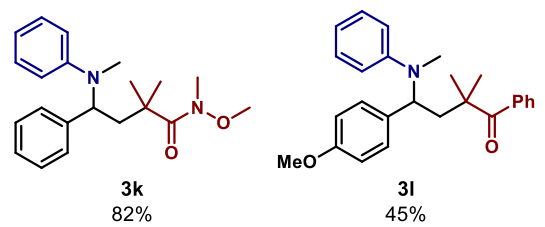

$\mathbf{3 k}$
$82 \%$

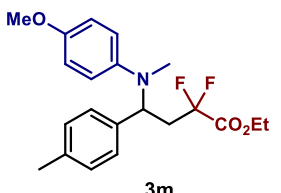

$3 m$
$42 \%$

${ }^{a}$ See SI for experimental details.

Figure 3. Mechanistic hypothesis for three-component alkene carbofunctionalization.

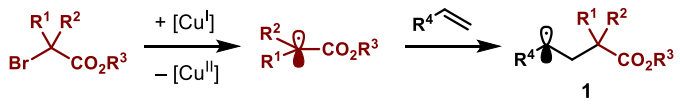

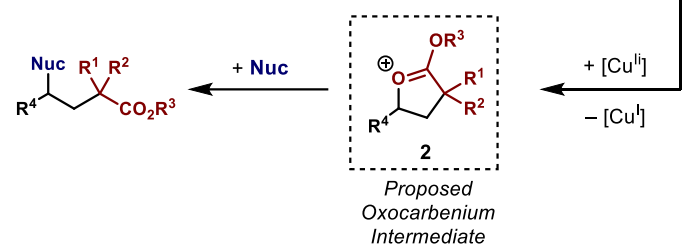

Our mechanistic hypothesis (Figure 3) involves the addition of electrophilic carbon-centered radicals to alkenes, generating radical addition intermediate $\mathbf{1}$. Subsequent oxidation by $\mathrm{Cu}(\mathrm{II})$ affords cyclic oxocarbenium ion $\mathbf{2}$. In the presence of secondary amines (Nuc $=\mathrm{HNR}_{2}$ ), carboamination results from nucleophilic ring opening of $\mathbf{2}$. We hypothesized that the catalytic generation of $\mathbf{2}$ could serve as a more general manifold for alkene carboheterofunctionalization with other classes of nucleophiles. Herein, we present our efforts to generalize this reaction platform: successful development of a new carboetherification, carboesterification, and carboarylation reactions are described. Further, we present mechanistic observations that provide an understanding of the factors that dictate the reactivity of the oxocarbenium intermediate.

\section{- RESULTS AND DISCUSSION}

Mechanistic observations: We have previously reported the development of a three-component alkene carboamination reaction that provides broad access to $\gamma$-aminocarbonyl compounds with secondary arylamine nucleophiles and iminolactones with primary amines. ${ }^{7 \text { a }}$ A summary of this reaction scope is provided in Table $\mathbf{1}$. Of note, both electron-poor (3b and $\mathbf{3 c}$ ) and electron-rich $(\mathbf{3 g}, \mathbf{3 h}$, $\mathbf{3 j}$, and 3l) alkenes undergo carboamination with this system, and several classes of activated alkyl bromides are suitable electrophiles (3j-3o). In general, both secondary arylamines and electronpoor primary arylamines yield $\gamma$-amino carbonyl compounds, an important class of compounds that are well-represented in biologically active molecules (Figure 2B).

When electron-neutral or -rich primary arylamines are used in this system, iminolactone structures result (Table 2). ${ }^{9}$ Several classes of aliphatic alkenes are readily converted to iminolactones under these conditions, including terminal aliphatic (5a), 1,1-disubstituted (5e and $\mathbf{5 f}$ ), and internal and unactivated alkenes (5g and 5h). Notably, both $(E)$ - and $(Z)-\beta$-methylstyrene converge upon the same iminolactone diastereomer with high diastereoselectivity for the trans product.

Table 2. Summarized scope of iminolactonization. ${ }^{a}$

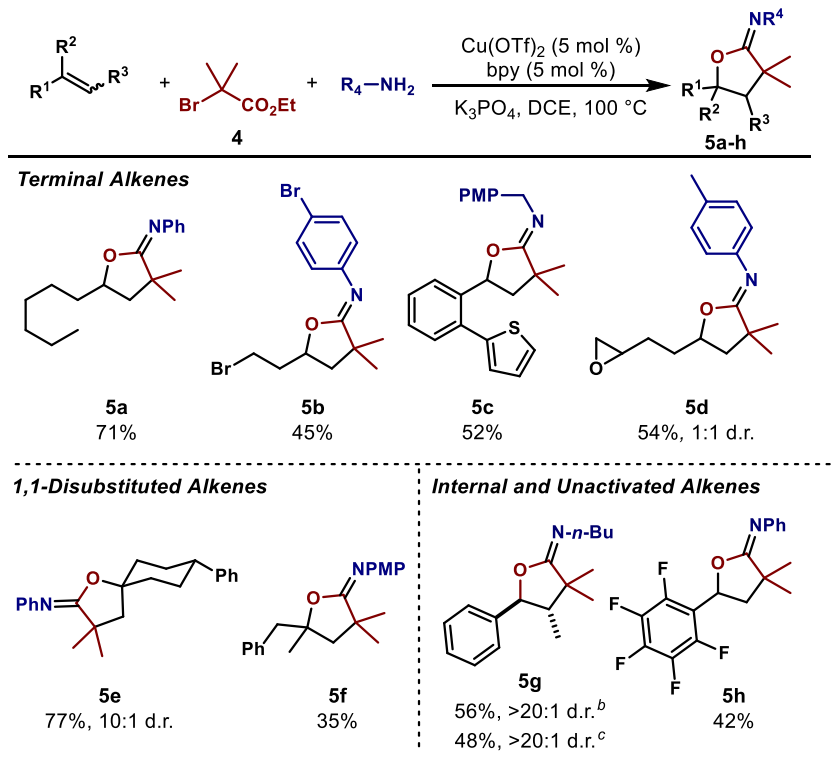

${ }^{a}$ See SI for experimental details. ${ }^{b}$ From $(Z)$-alkene. ${ }^{c}$ From $(E)$ - alkene. 


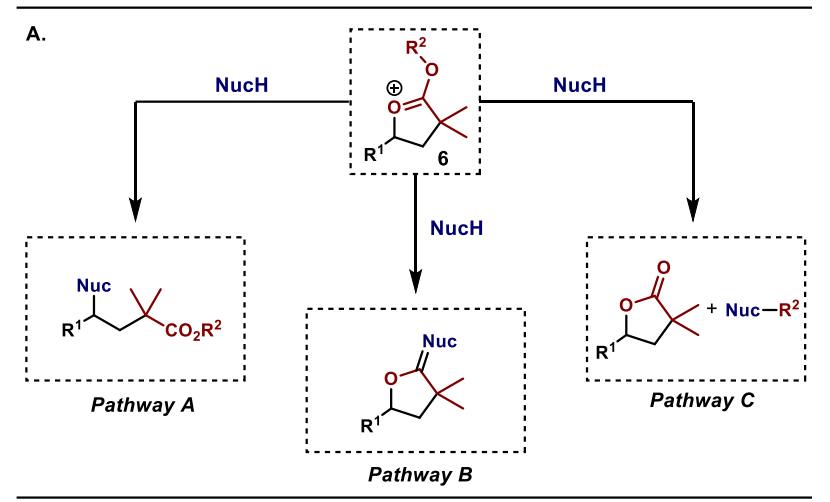

B. Secondary Amines - Pathway A

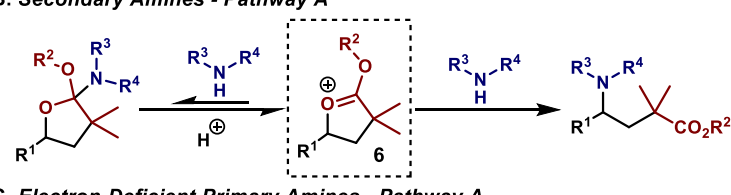

C. Electron-Deficient Primary Amines - Pathway A

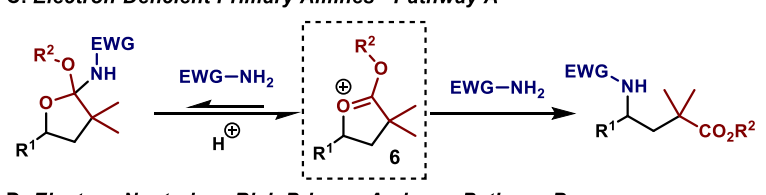

D. Electron-Neutral or -Rich Primary Amines - Pathway $B$

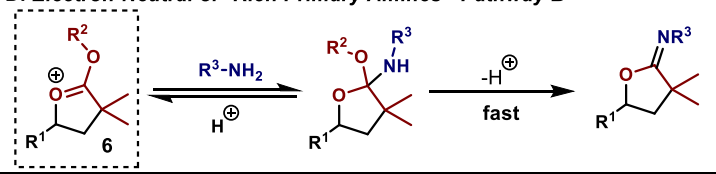

Figure 4. The degree of substitution as well as the electronic nature of the amine nucleophile influence product selectivity

We posit that the mechanistic divergence between primary and secondary amines is consistent with the proposed intermediate 6, which could react with a nucleophile via several distinct pathways (Figure 4, A). The specific pathway expected to be operative with a given combination of reaction components must depend on both the steric and electronic properties of $\mathbf{6}$ as well as the nature of the nucleophile. Secondary and/or electron-deficient amines likely undergo reversible attack at the carbonyl position resulting ultimately in irreversible nucleophilic ring opening via pathway A (Figure 4, B and C). Electron-rich primary amines, however, undergo condensation onto the oxocarbenium to yield iminolactones via pathway B (Figure 4, D). Evidence of the intermediacy of 6 is present in several observations. When the standard ethyl ester is replaced with $t$-butyl ester $\mathbf{7}$ and combined with electronically differentiated vinylarenes, lactones $\mathbf{8 a - 8 b}$ are formed selectively, and transfer of the $t$-butyl group to the nitrogen nucleophile to form $\mathrm{N}$ methyl- $N$-tert-butyl- $p$-toluidine is observed by GCMS (Scheme 1). ${ }^{10}$

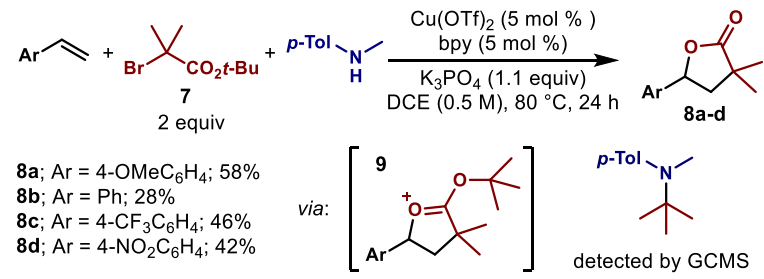

Scheme 1. Lactone formation observed with tert-butyl substituted haloester.

We propose that these lactone structures result from the ionization of intermediate 9. Notably, lactonization is observed even with electron rich vinylarenes, indicating oxocarbenium intermediates are operative with substrates possessing alternative cation-stabilizing groups (e.g., an anisyl fragment, 8a).

Although the formation of 8a-d is consistent with an oxocarbenium as an intermediate in this system, it does not provide information about the nature of the oxidation event that precedes its formation. Although work to elucidate the mechanism of this oxidation is presently underway, we cannot at present ascribe a specific pathway. Furthermore, it is possible that vinylarenes and aliphatic alkene substrates undergo different oxidation mechanisms. Some observational evidence does suggest that atom transfer radical addition (ATRA) intermediates could precede oxocarbenium formation with aliphatic alkenes. For instance, when $\mathbf{1 0}$ is combined with 11, only ATRA adduct 12 is observed (Scheme 2). Intermediate 13 en route to this product differs from previously discussed radical addition intermediates in that it lacks the ThorpeIngold effect (TIE) that is expected to enhance the interaction of the secondary radical with the ester moiety. This reduced interaction could inhibit the ability of the $\mathrm{Cu}$ (II) catalyst to oxidize the radical to the

Scheme 2. ATRA adduct resulting from secondary haloester

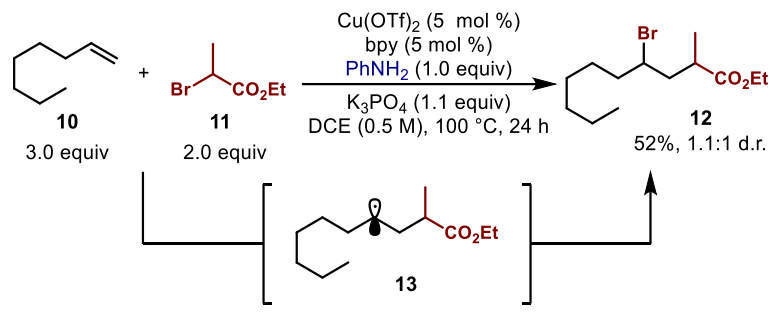

oxocarbenium in favor of direct bromine atom transfer. Alternatively, the same reduced TIE could prevent intramolecular displacement of the bromide by the ester moiety in 13. ATRA products also result from alkene substrates that prevent involvement of the ester group by other means; thus, $\mathbf{1 4}$ selectively yields pyrrolidine 15 (Scheme 3). In this case, addition of the alkyl radical initiates intramolecular cyclization, which transposes the radical to a position more distal to the ester moiety (16). Consequently, ATRA predominates.

Scheme 3. ATRA adduct resulting from $N, N$-diallylaniline radical trap.

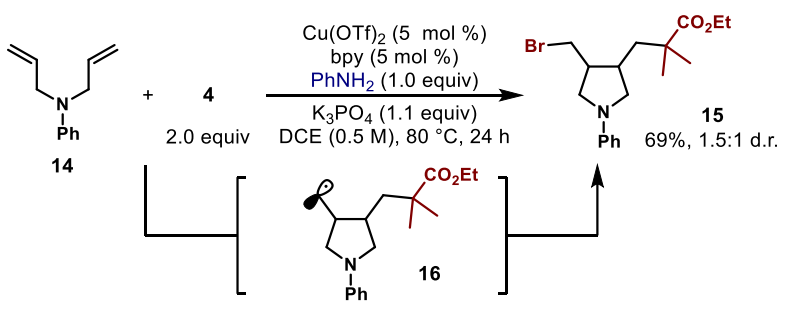

As both $\mathbf{1 0}$ and $\mathbf{1 4}$ are terminal aliphatic alkene substrates, generalizations about ATRA intermediates for all classes of alkenes cannot be asserted.

During reaction development, it was noted that the difunctionalization of aliphatic alkenes was restricted to the use of primary amines, as secondary nucleophiles yielded only lactones and the corresponding alkylated amine (Scheme 4). Intermediate 19, which readily undergoes condensation with primary amines to produce iminolactones (Table 2, 5a), instead reacts at the primary alkyl position of the ethyl ester when paired with a secondary arylamine to produce 18 (Figure 4, pathway C). This represents a distinction between the reactivity of vinylarenes and aliphatic alkenes, the former family of substrates generally reacting with secondary amines to yield carboamination products. Intermediate $\mathbf{1 9}$ is distinct from 
oxocarbeniums generated from vinylarenes, in that neither of the electrophilic moieties competing for nucleophilic attack are activated benzylic positions. We propose, therefore, that dealkylation through attack of the more reactive primary position predominates when aliphatic alkenes are paired with secondary arylamines.

Scheme 4. Lactone production from aliphatic alkene and secondary arylamine (Pathway C, Figure 4).

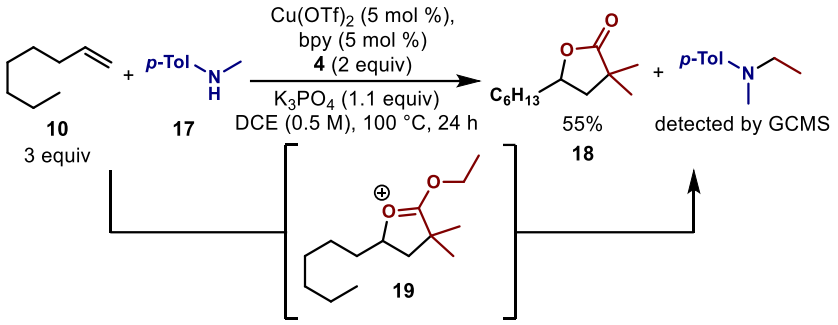

Given this hypothesis, we sought to ameliorate this scope limitation by employing neopentyl ester 20 instead of 4 (Table 3). This electrophile is expected to produce intermediate 22, which would no longer be susceptible to the dealkylation reactivity that prevented amine incorporation in 19. Additionally, 22 would not be susceptible to fragmentation as $\mathbf{9}$ is. Gratifyingly, this modified electrophile enabled access to a selection of carboamination products derived from secondary arylamines and 1-octene (21a-c) which could not be previously prepared. This finding validates the notion that the steric and electronic properties of 2 (Figure 4), combined with the characteristics of the nucleophile, together dictate which mechanistic pathway is traversed. By altering the identity of $\mathrm{R}^{2}$ (Figure 4, structure 6), pathway $\mathrm{C}$ is closed, and pathway $\mathrm{A}$ becomes dominant.

Table 3. Carboamination of aliphatic alkenes enabled by neopentyl-substituted electrophile (Pathway A, Figure 4).
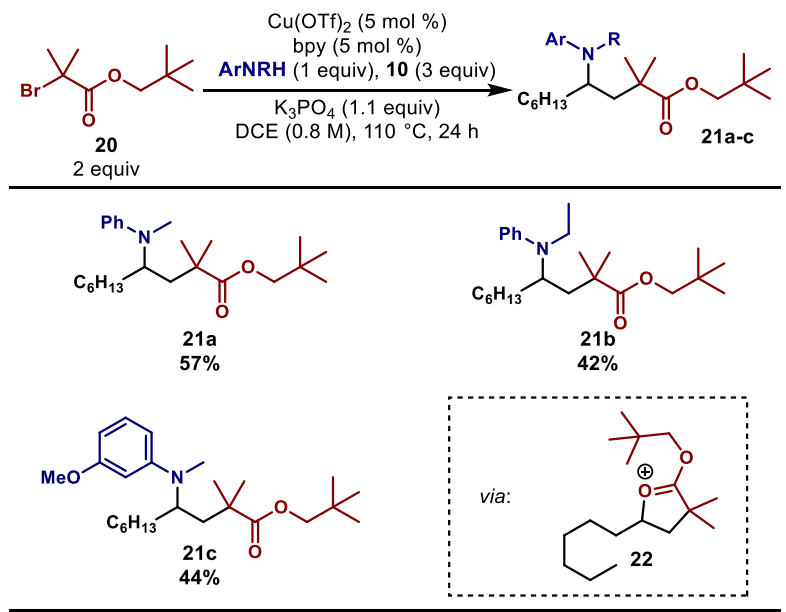

Beyond the influence that the oxocarbenium intermediate exerts upon chemoselectivity, it is also clear that this structure should be reconcilable with the high degree of diastereoselectivity observed when employing internal acyclic alkenes. As depicted in Table 2, the use of most primary arylamines results in the generation of iminolactones. The formation of $\mathbf{2 4}$ in high d.r. from alkene 23 has already been established (Figure 5, A) ${ }^{7 \mathrm{a}}$ This observation is consistent with condensation of the amine nucleophile onto a trans oxocarbenium intermediate $\mathbf{2 5}$.
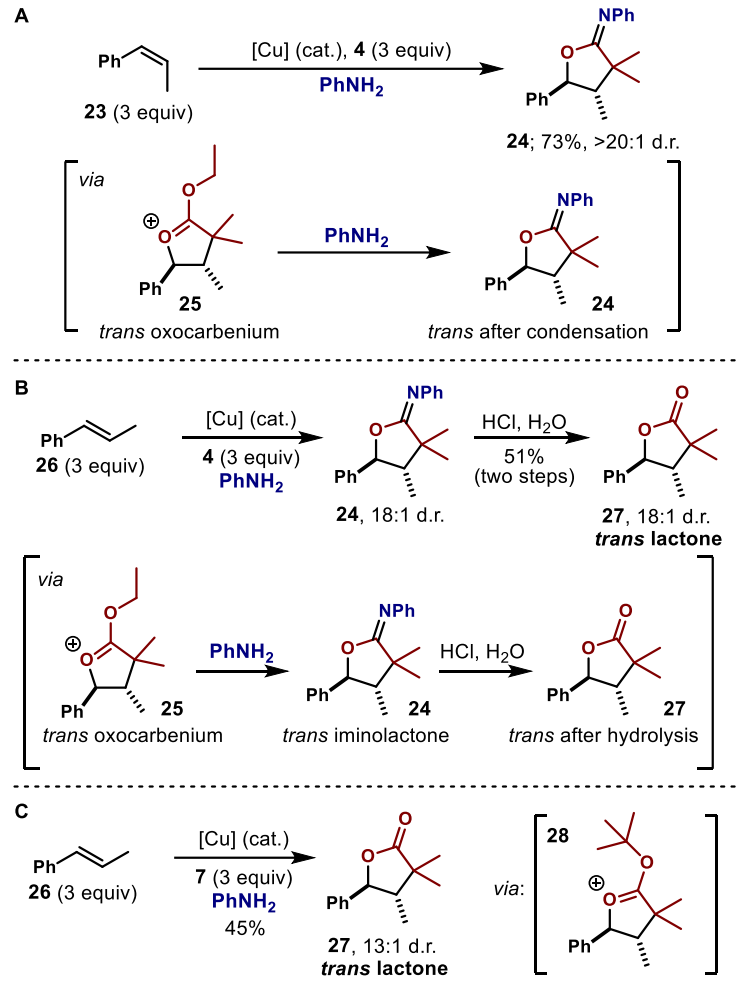

Figure 5: A: Iminolactone stereochemistry consistent with formation of trans oxocarbenium. B: Iminolactone $\mathbf{2 4}$ hydrolyzes cleanly to trans lactone $\mathbf{2 7}$. C: trans-lactone $\mathbf{2 7}$ is also accessible via use of 7, as expected from proposed oxocarbenium $\mathbf{2 8}$.

The diastereoconvergence of this reaction permits access to trans configured products from either the $E$ or $Z$ olefin (or mixtures); thus, 26 yields the same diastereomer as that which results from use of 23 (Figure 5, B). Hydrolysis of this iminolactone product yields clean access to the corresponding trans lactone 27. As expected, an identical lactone can be produced by employing the same trapping strategy outlined in Scheme 1 via tert-butyl haloester 7 (Figure 5, C). These outcomes together support the notion that the stereochemistry of the oxocarbenium intermediate is retained in the products.

Although stereodefined iminolactones result from the combination of internal alkenes and primary arylamine nucleophiles (Figure 4, pathway B), this is contingent upon the electronic properties of the alkene component. When $\mathbf{2 9}$, administered as a mixture of isomers, is combined with a primary arylamine, the acyclic carboamination product $\mathbf{3 0}$ is formed as the major product and as a single diastereomer (Scheme 5). While the highly electron-rich nature of 29 could be expected to facilitate $\mathrm{C}-\mathrm{N}$ bond formation via a mechanism involving purely acyclic carbocation intermediates, the high degree of diastereoselectivity is suggestive of a high degree of conformational control in the intermediates leading to $\mathbf{3 0}$.

Scheme 5: Electron-rich 29 yields cis $\gamma$-lactam when paired with primary arylamine. See SI for details. 


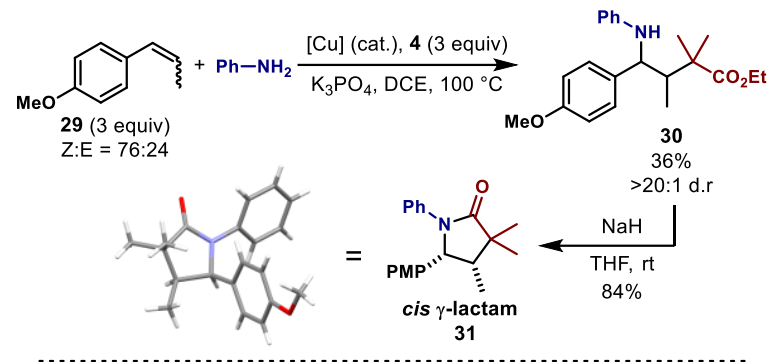

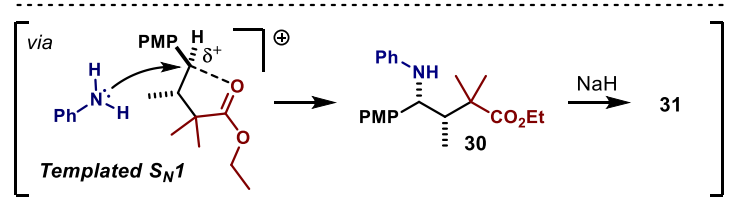

Treatment of $\mathbf{3 0}$ with sodium hydride elicits clean conversion to cis $\gamma$-lactam 31 as a single isomer, with $\mathrm{x}$-ray crystallography confirming both connectivity and stereochemistry. This stereochemical outcome is consistent with the mechanistic hypothesis outlined in Figure 4, pathway A, which has heretofore been attributed to the intermediacy of $\mathbf{6}$. This exact mechanism is unlikely, however, as iminolactone formation is expected to occur from this intermediate in the presence of an electron-neutral primary arylamine (and is readily observed with other internal alkenes (Figure 5, A)). Rather, it appears that $\mathbf{3 0}$ is obtained from an intermediate mechanism involving a conformationally rigid intermediate structure that does not activate the carbonyl group toward iminolactonization. Such stereoinvertive $S_{N} 1$ reactions are precedented in the literature, and it is proposed that $\mathbf{3 0}$ is obtained through such a pathway. ${ }^{11}$

While a primary arylamine paired with 37 results in two-step access to the cis $\gamma$-lactam 31, aliphatic amines combine with 29 to produce trans lactam structures in a single step. Thus, benzylamine yields trans $\gamma$-lactam 32 directly in >20:1 d.r. (Scheme 6). In this case, the lactam structure could be obtained from either of the pathways outlined in Figure 4 (pathway A or B), with equilibration through the acyclic carbocation $\mathbf{3 3}$ likely funneling material to the thermodynamically preferred trans $\gamma$-lactam structure. Importantly, this isomerization pathway is apparently inaccessible to iminolactone structures derived from less electron-rich olefin components (Figure 5) and to 30, which failed to cyclize and thus be subject to equilibration.

Scheme 6: Electron-rich 29 yields trans $\gamma$-lactam when paired with primary aliphatic amine (Pathway A and/or B, Figure 4). See SI for details.

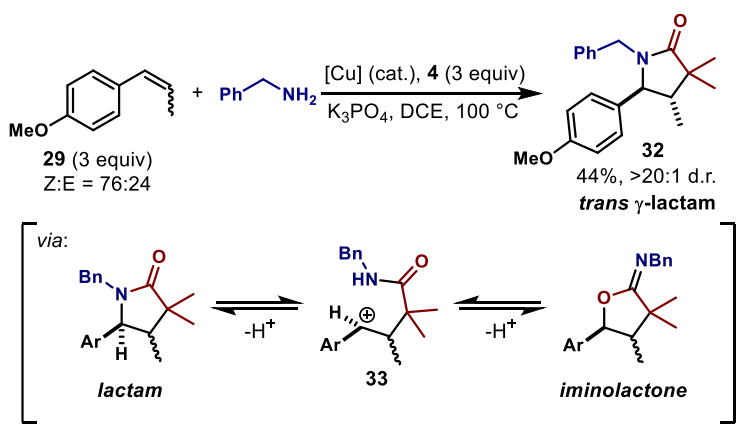

Expansion of synthetic utility: Although 29 does provide access to $\gamma$-lactams in a single step, we sought to establish a more general protocol for accessing such structures, given their prevalence in bioactive compounds (Figure 2, B). Toward this end, we were delighted to find that benzophenone imine (34) readily functions as a nucleophile, providing carboimination product $\mathbf{3 5}$ in excellent yield (eq. 1).

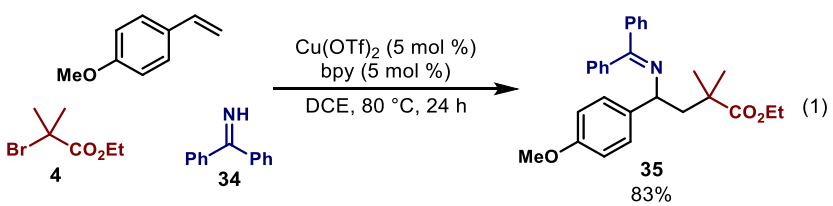

The success of $\mathbf{3 4}$ as a nucleophile in this carbofunctionalization system represents an opportunity to rapidly access valuable $\gamma$ lactam structures applying a subsequent hydrolysis/lactamization sequence. To demonstrate the viability of this route, a telescoped reaction sequence was developed (Table 4). Electron-neutral (36a) and -rich (36b and 36c) vinylarenes participate in the tandem reaction sequence to afford $\gamma$-lactam products in moderate yields, including those possessing heterocyclic functionality $(\mathbf{3 6 c})$. An acetal-bearing vinylarene produced a fair yield of the lactam product (36d).

Table 4. Access to $\gamma$-Lactam structures via tandem carboamination/lactamization. ${ }^{a}$

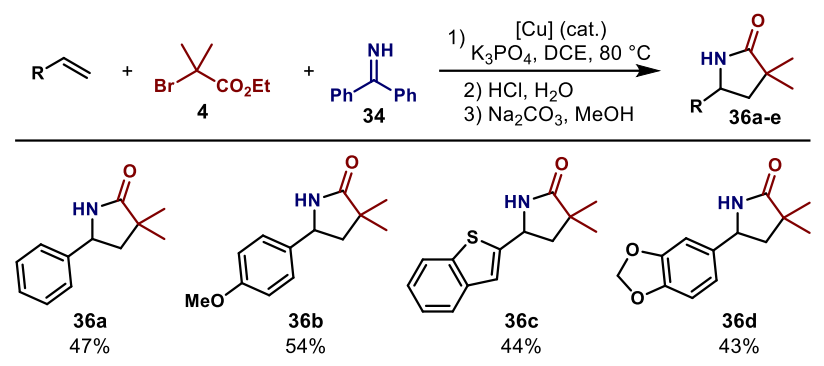

${ }^{a}$ See SI for experimental details.

The mechanistic hypothesis for this alkene carboamination reaction suggests that the amine is primarily a bystander that intercepts catalytically generated $\mathbf{2}$ (Figure 3 ) to afford amination products. This indicates that this system could be suitable as a general alkene carboheterofunctionalization platform with diverse classes of nucleophiles. Initial experimentation toward this end commenced with the substitution of the arylamine with phenol nucleophiles. The combination of styrene (37), ethyl 2-bromoisobutyrate (4), and 4-methoxyphenol (38) under these conditions yield the carboetherification product 39a in a promising in situ yield of $25 \%$ (eq. 2). Significant quantities of byproducts were observed under these conditions, including the alkylated olefin $\mathbf{4 0}$ and lactone $\mathbf{4 1}$ in $12 \%$ and $41 \%$ in situ yields, respectively.

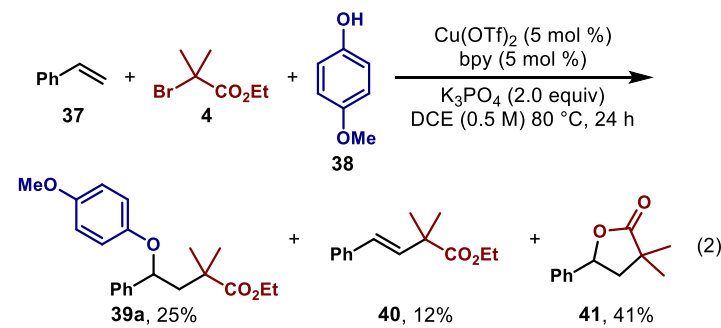

${ }^{a}$ In situ yields determined by GC analysis.

Initial experimentation sought to improve the yield of 39a by increasing the loading of $\mathbf{3 7}$. It was found that an excess of styrene improves the yield of 39a, albeit with little impact on the quantities of $\mathbf{4 0}$ and 41 (Table 5, entries 1-3). Given that the byproducts 40 and $\mathbf{4 1}$ likely arise from elimination processes of cationic intermediates, and that phenols and arylamines differ considerably in $\mathrm{pKa}$, a survey of bases was conducted (Table 5, entries 4-9). 
Table 5. Selected optimization results for three-component alkene carboetherification $^{a}$

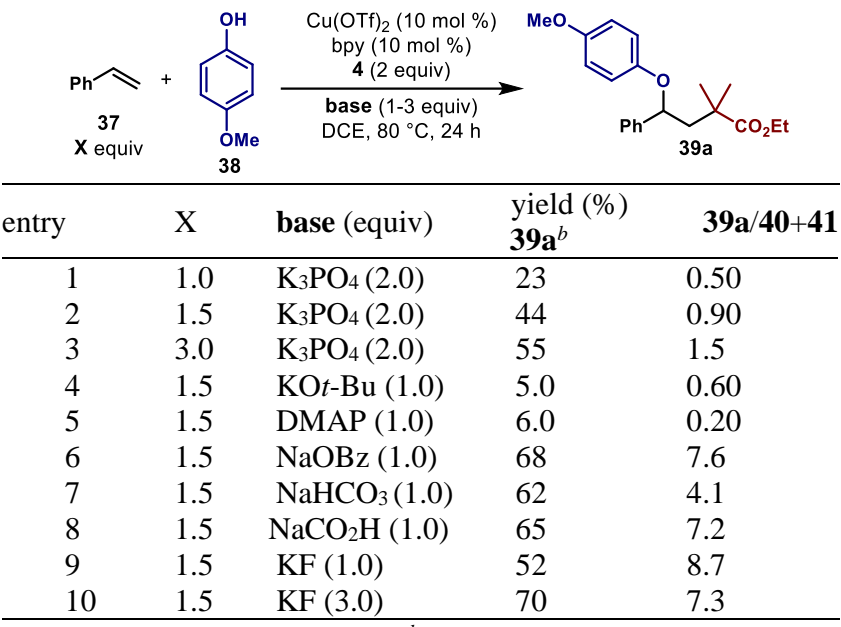

${ }^{a}$ See SI for experimental details. ${ }^{b}$ Yield determined by GC analysis.

Potassium fluoride (KF) was found to be particularly effective at increasing the yield of 39a while simultaneously suppressing the formation of byproducts $\mathbf{4 0}$ and $\mathbf{4 1}$ (Table 5, entry 9). Further modifications to the KF stoichiometry led to the optimized conditions (Table 5, entry 10): 1.0 equivalent of the aryl alcohol, 1.5 equivalents of the alkene, and 2.0 equivalents of the $\alpha$-haloester can be combined in the presence of $10 \mathrm{~mol} \% \mathrm{Cu}(\mathrm{OTf})_{2}, 10 \mathrm{~mol} \% 2,2-$ bipyridine (bpy), and 3.0 equivalents of KF to furnish the model carboetherification product 39a in $70 \%$ isolated yield.

Having established optimized conditions for the carboetherification of styrene with aryl alcohols, we began a broader investigation of the reaction scope (Table 6). Electron neutral and rich phenols are amenable in this system, providing good yields of the products (39a-c). Mildly electron poor phenols bearing $\mathrm{F}, \mathrm{Cl}, \mathrm{Br}$, or I substituents can also be applied (39d-g), demonstrating that halide functionalities common in traditional cross-coupling and $\mathrm{S}_{\mathrm{N}} \mathrm{Ar}$ reactions are well tolerated under the reaction conditions.

Despite the lower nucleophilicity of phenols as compared to amines, even electron poor derivatives are found to participate readily in the reaction. Both ethylparaben and a 3-(trifluoromethyl)phenol are converted to products $39 \mathbf{h}$ and $39 \mathbf{i}$, respectively, in synthetically useful yields. Probing the limits of this electronic tolerance, 4-nitrophenol is converted to product $39 \mathbf{j}$ in excellent $85 \%$ yield. Pentafluorophenol was found to be an ineffective nucleophile under the standard conditions, however changing the base from KF to triethylamine restores reactivity and affords $39 \mathrm{k}$ in $70 \%$ yield. Several phenols bearing ortho substitution were also examined, providing insight into the steric limitations of the reaction. Notably, both 2-cyanophenol and 2-phenylphenol react to produce good yields of 391 and $39 \mathrm{~m}$, respectively. An electron rich olefin, 4-vinylanisole, when paired with a modestly electron poor nucleophile, 4-chlorophenol, produces a good yield of carboetherification product 39n.

Combining 4-methoxyphenol and electron poor 4-(trifluoromethyl)styrene results in the formation of $\mathbf{3 9 o}$ in modest yield.

Table 6. Scope of three-component alkene carboetherification with phenols ${ }^{a}$

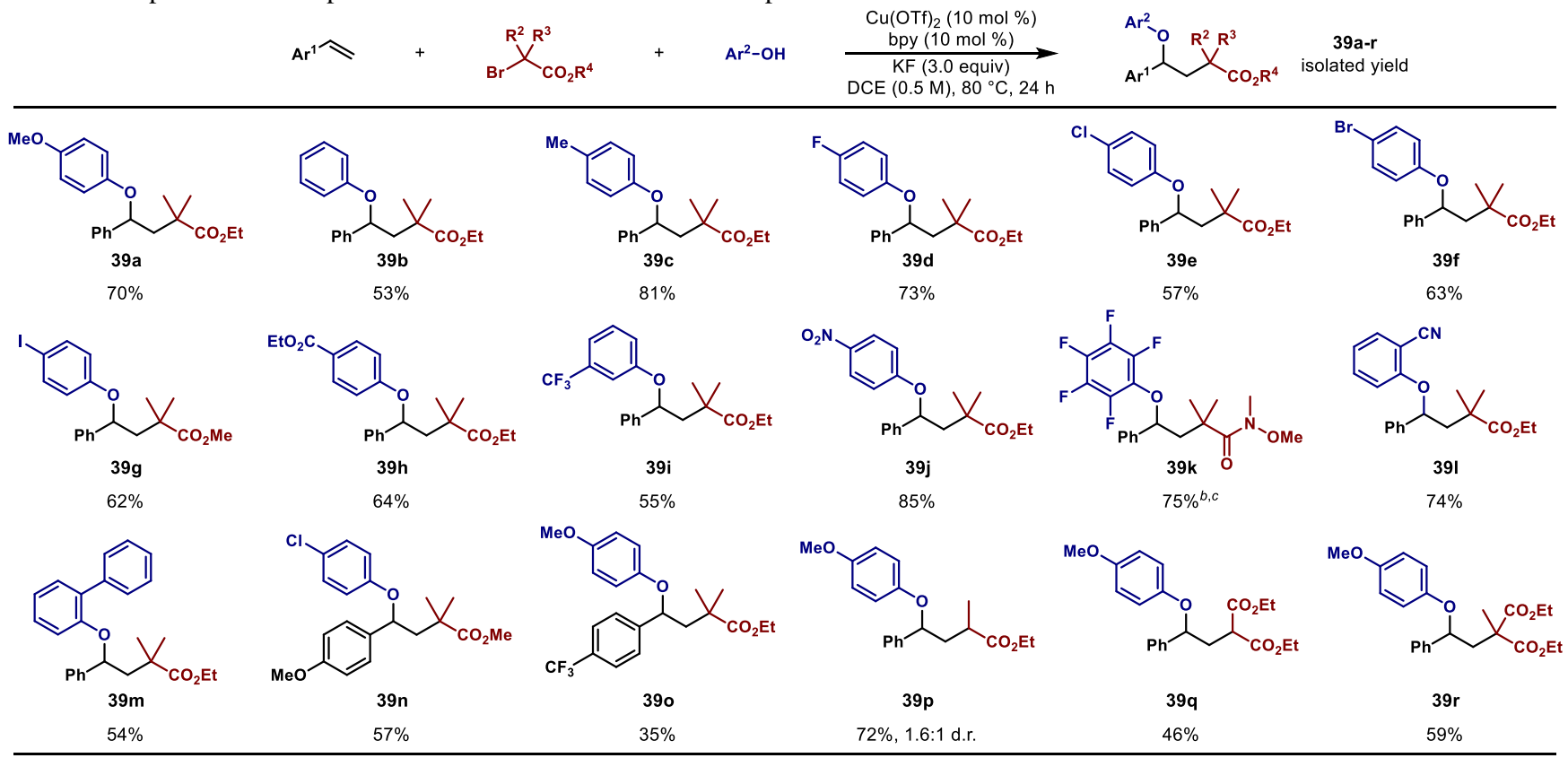

${ }^{a}$ See SI for experimental details. ${ }^{b}$ Triethylamine (2.0 equiv) used instead of KF. ${ }^{c}$ PMDTA $(N, N, N, N$ ', $N$ '-pentamethyldiethylenetriamine, 10 mol \%) used instead of bpy.

When ethyl 2-bromopropionate is used, a good yield of 39p is obtained with low diastereoselectivity $($ d.r. $=1.6: 1)$. Malonate-derived alkyl bromides are also viable electrophilic components in this system, as diethyl bromomalonate and diethyl 2-bromo-2methylmalonate undergo conversion to $\mathbf{3 9 q}$ and $39 \mathbf{r}$, respectively, in moderate yields. Such electrophiles are useful for further synthetic elaboration of the product carbonyl groups, or, alternatively, for obtaining the less substituted products via known decarboxylation protocols. ${ }^{12}$

During efforts to optimization the alkene carboetherification reaction with phenol nucleophiles, it was noted that carboxylate bases, despite being competent promoters of the desired reaction (Table 5 , entries 6 and 8), were capable as serving as nucleophiles to form carboesterification products. As the intentional use of carboxylate nucleophiles would entail a straightforward means of 
accessing protected benzylic alcohols, we sought optimized conditions for this class of nucleophile. Although merely conducting the reaction in the absence of phenol yielded none of the desired carboesterification adduct 42a (Scheme 7, A), it was found that replacement of the standard $\mathrm{Cu}(\mathrm{OTf})_{2}$ salt with $\left[\mathrm{Cu}(\mathrm{MeCN})_{4}\right] \mathrm{PF}_{6}$ delivers 42a in 65\% yield (Scheme 7, B). Additionally, it proved possible to substitute potassium benzoate for the parent benzoic acid, provided that potassium phosphate was employed as an added base (Scheme 7, C). Thus, acyl fragments can be readily installed with either the potassium salt or the free acid, depending on the needs of the practitioner.

Scheme 7. Reactivity with benzoate nucleophiles in absence of phenol
A

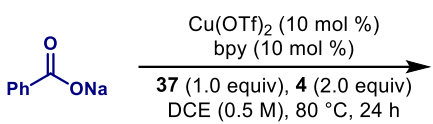

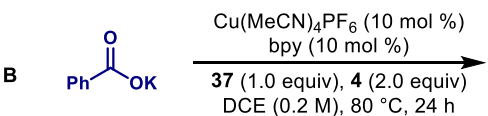

$\mathrm{Cu}(\mathrm{MeCN})_{4} \mathrm{PF}_{6}(10 \mathrm{~mol} \%)$ bpy $(10 \mathrm{~mol} \%)$

c $\stackrel{\mathrm{Ph}}{\mathrm{OH}}_{\mathrm{OH}}$
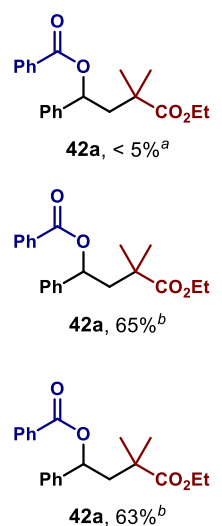

${ }^{a}$ In situ yield determined by GC analysis. ${ }^{b}$ Isolated yield.

An assessment of basic salts as nucleophiles demonstrates the ability to install a variety of common protecting groups into the substrate (Table 7). Both benzoate (42a) and acetate (42b) are viable nucleophiles in this three-component reaction to afford a variety of diester products. Importantly, the selective deprotection of the less hindered benzylic ester moiety is known, providing a means to chemoselectively elaborate this class of diester products. ${ }^{13}$ Further evaluation of other nucleophile classes revealed that electron rich aryl rings participate in the reaction, delivering diversely functionalized 1,1-diaryl alkane structures (42c-e) with only slight modifications to the standard reaction conditions.

Table 7. Additional oxygen and carbon nucleophiles participate in three-component alkene carbofunctionalization ${ }^{a}$

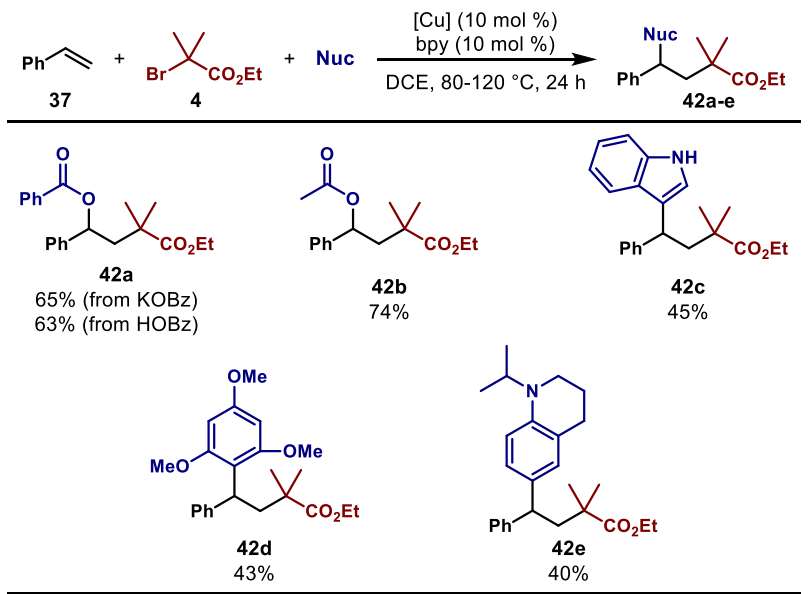

${ }^{a}$ See SI for experimental details.

\section{- CONCLUSIONS}

In summary, we have developed a three-component carbofunctionalization system that converts diverse classes of nucleophiles, alkenes, and activated alkyl halides to the corresponding carboheterofunctionalization products. Our observations suggest that the reaction proceeds through a 5-membered oxocarbenium ion intermediate. When paired with electron-neutral or poor alkenes, primary arylamines produce iminolactones. When paired with secondary arylamines, phenols, or other nucleophiles, all vinylarenes result in $\gamma$-functionalized carbonyl compounds. Electronrich alkenes, when paired with primary amines, convert directly to the corresponding $\gamma$-lactam structures. Internal alkenes undergo conversion to the corresponding carbofunctionalization products with high diastereoselectivity in all cases examined. Efforts to understand the mechanism of radical oxidation preceding oxocarbenium formation are ongoing and will be reported in due course.

\section{- ASSOCIATED CONTENT}

Accession Codes

CCDC 2071439 contains supplementary crystallographic data. Information accessible from www.ccdc.cam.ac.uk/data_request/cif, or by emailing data_request@ccdc.cam.ac.uk, or by contacting The Cambridge Crystallographic Data Centre, 12 Union Road, Cambridge CB2 1EZ, UK; fax: +44 1223336033.

\section{- AUTHOR INFORMATION}

\section{Corresponding Author}

Kami. L. Hull - University of Texas at Austin, Department of Chemistry, Austin, Texas 78712, United States; orcid.org/00000003-3102-2686; Email: kamihull@utexas.edu

\author{
Authors \\ Travis L. Buchanan - ORCID: 0000-0001-9038-9790 \\ Samuel N. Gockel - ORCID: 0000-0003-3584-066X \\ Alexander M. Veatch - ORCID: 0000-0002-7564-8755 \\ Ya-Nong Wang - ORCID: 0000-0002-5838-3855
}

Notes

The authors declare no competing financial interests.

\section{- ACKNOWLEDGMENTS}

The authors would like to thank Prof. Katie Mitchell-Koch and Mr. Ryan Steinert for preliminary calculations and helpful discussions. They would also like to thank the NIH NIGMS, the Welch Foundation, the Novartis, Eli Lilly and Co., as well as the University of Texas at Austin for their generous support.

\section{- REFERENCES}

(1) For selected one-component alkene carboamination examples: (a) Wdowik, T.; Galster, S. L.; Carmo, R. L. L.; Chemler, S. R. Enantioselective, Aerobic Copper-Catalyzed Intramolecular Carboamination and Carboetherification of Unactivated Alkenes. ACS Catal. 2020, 10, 8535-8541. (b) Miao, L.; Haque, I.; Manzoni, M. R.; Tham, W. S.; Chemler, S. R. Diastereo- and Enantioselective Copper-Catalyzed Intramolecular Carboamination of Alkenes for the Synthesis of Hexahydro-1H-benz[f]indoles. Org. Lett. 2010, 12, 4739-4741. (c) He, W.; Yip, K.-T.; Zhu, N.-Y.; Yang, D. Pd(II)/tBu-quinolineoxazoline: An Air-Stable and Modular Chiral Catalyst System for Enantioselective Oxidative Cascade Cyclization. Org. Lett. 2009, 11, 5626-5628. (d) Zeng, W.; Chemler, S. R. Copper(II)-Catalyzed Enantioselective Intramolecular Carboamination of Alkenes. J. Am. Chem. Soc. 2007, 129, 12948-12949. Selected two-component carboamination examples: (e) 
Ozols, K.; Onodera, S.; Wozniak, L; Cramer, N. Cobalt(III)-Catalyzed Enantioselective Intermolecular Carboamination by C-H Functionalization. Angew. Chem. Int. Ed. 2021, 60, 655-659. (f) Duchemin, C.; Cramer, N. Enantioselective $\mathrm{Cp}^{\mathrm{x}} \mathrm{Rh}^{\mathrm{III}}$-Catalyzed Carboaminations of Acrylates. Angew. Chem. Int. Ed. 2020, 59, 14129-14133. (g) He, J.; Xue, Y.; Han, B.; Zhang, C.; Wang, Y.; Zhu, S. Nickel-Catalyzed Asymmetric Reductive 1,2-Carboamination of Unactivated Alkenes. Angew. Chem. Int. Ed. 2020, 59, 2328-2332. (h) Bao, X.; Wang, Q.; Zhu, J. Palladium-Catalyzed Enantioselective Narasaka-Heck Reaction/Direct C-H Alkylation of Arenes: Iminoarylation of Alkenes. Angew. Chem. Int. Ed. 2017, 56, 9577-9581. (i) Garlets, Z. J.; White, D. R.; Wolfe, J. P. Recent Developments in $\mathrm{Pd}^{0}$-Catalyzed Alkene-Carboheterofunctionalization Reactions. Asian J. Org. Chem. 2017, 6, 636653. (j) Lerchen, A.; Knecht, T.; Daniliuc, C. G.; Glorius, F. Unnatural Amino Acid Synthesis Enabled by the Regioselective Cobalt(III)Catalyzed Intermolecular Carboamination of Alkenes. Angew. Chem. Int. Ed. 2016, 55, 15166-15170. (k) Hu, Z.; Tong, X.; Liu, G. Rhodium(III) Catalyzed Carboamination of Alkenes Triggered by $\mathrm{C}-\mathrm{H}$ Activation of $\mathrm{N}$-Phenoxyacetamides Under Redox-Neutral Conditions Org. Lett. 2016, 18, 1702-1705. (1) Um, C.; Chemler, S. R. Synthesis of 2-Aryl- and 2-Vinylpyrrolidines via Copper-Catalyzed Coupling of Styrenes and Dienes with Potassium $\beta$-Aminoethyl Trifluoroborates. Org. Lett. 2016, 18, 2515-2518. (m) Piou, T.; Rovis, T. Rhodium-Catalyzed syn-Carboamination of Alkenes via a Transient Directing Group. Nature, 2015, 527, 86-90. (n) Faulkner, A.; Scott, J. S.; Bower, J. F. An Umpolung Approach to Alkene Carboamination: Palladium Catalyzed 1,2-Amino-Acylation, -Carboxylation, -Arylation, -Vinylation, and -Alkynylation. J. Am. Chem. Soc. 2015, 137, 7224-7230. (o) Hopkins, B. A.; Wolfe, J. P. Synthesis of Enantiomerically Enriched Imidazolidin-2-ones through Asymmetric Palladium-Catalyzed Alkene Carboamination. Angew. Chem. Int. Ed. 2012, 51, 9886-9890. (p) Tkatchouk, E.; Mankad, N. P.; Benitez, D.; Goddard, W. A.; Toste, F. D. Two Metals are Better than One in the Gold Catalyzed Oxidative Heteroarylation of Alkenes. J. Am. Chem. Soc. 2011, 133, 1429314300. (q) Ney, J. E.; Wolfe, J. P. Palladium-Catalyzed Synthesis of $N$ Aryl Pyrrolidines from $\gamma$-( $N$-Arylamino $)$ Alkenes: Evidence for Chemoselective Alkene Insertion into Pd-N Bonds. Angew. Chem. Int. Ed. 2004, 43, 3605-3608.

(2) Selected one-component carboetherification examples: (a) Karyakarte, S. D.; Um, C.; Berhane, I. A.; Chemler. S. R. Synthesis of Spirocyclic Ethers by Enantioselective Copper-Catalyzed Carboetherification of Alkenols. Angew. Chem. Int. Ed. 2018, 57, 12921-12924. (b) Miller, Y.; Miao, L.; Hosseini, A. S.; Chemler, S. R. Copper-Catalyzed Intramolecular Alkene Carboetherification: Synthesis of Fused-Ring and Bridged-Ring Tetrahydrofurans. J. Am. Chem. Soc. 2012, 134, 12149-12156. (c) Nakhla, J. S.; Kampf, J. W.; Wolfe, J. P. Intramolecular Pd-Catalyzed Carboetherification and Carboamination. Influence of Catalyst Structure on Reaction Mechanism and Product Stereochemistry. J. Am. Chem. Soc. 2006, 128, 2893-2901. Selected two-component carboetherification: (d) Tsui, E.; Metrano, A. J.; Tsuchiya, Y.; Knowles, R. R. Catalytic Hydroetherification of Unactivated Alkenes Enabled by Proton-Coupled Electron Transfer. Angew. Chem. Int. Ed. 2020, 59, 11845-11849. (e) Silva, A. R.; Polo, E. C.; Martins, N. C.; Correia, C. R. D. Enantioselective Oxy-HeckMatsuda Arylations: Expeditious Synthesis of Dihydrobenzofuran Systems and Total Synthesis of the Neolignan (-)-Conocarpan. Adv. Synth. Catal. 2018, 360, 346-365. (f) Chen. D.; Chemler, S. R. Synthesis of Phthalans via Copper-Catalyzed Enantioselective Cyclization/Carboetherification of 2-Vinylbenzyl Alcohols. Org. Lett. 2018, 20, 64536456. (g) Hu, N.; Li, K.; Wang, Z.; Tang, W. Synthesis of Chiral 1,4benzodioxanes and Chromans by Enantioselective Palladium-Catalyzed Alkene Aryloxyarylation Reactions. Angew. Chem. Int. Ed. 2016 , 55, 5044-5048. (h) Borrajo-Calleja, G. M.; Bizet, V.; Mazet, C. Palladium-Catalyzed Enantioselective Intermolecular Carboetherification of Dihydrofurans. J. Am. Chem. Soc. 2016, 138, 4014-4017. (i) Cahard, E.; Bremeyer, N.; Gaunt, M. J. Copper-Catalyzed Intramolecular Electrophilic Carbofunctionalization of Allylic Amides. Angew. Chem. Int. Ed. 2013, 52, 9284-9288. (j) Zhang, G.; Cui, L.; Wang, Y.; Zhang, L. Homogeneous Gold-Catalyzed Oxidative Carboheterofunctionalization of Alkenes. J. Am. Chem. Soc. 2010, 132, 1474-1475. (k) Wolfe, J. P.; Rossi, M. A. Stereoselective Synthesis of Tetrahydrofurans via the Palladium-Catalyzed Reaction of Aryl Bromides with $\gamma$-Hydroxy Alkenes: Evidence for an Unusual Intramolecular Olefin Insertion into a $\mathrm{Pd}(\mathrm{Ar})(\mathrm{OR})$ Intermediate. J. Am. Chem. Soc. 2004, 126, 1620-1621.

(3) Selected examples: (a) Li, Z.; Zhang, M.; Zhang, Y.; Liu, S.; Zhao, J.; Zhang, Q. Multicomponent Cyclopropane Synthesis Enabled by CuCatalyzed Cyclopropene Carbometalation with Organoboron Reagent: Enantioselective Modular Access to Polysubstituted 2-Arylcyclopropylamines. Org. Lett. 2019, 21, 5432-5437. (b) Puyl, V.; Derosa, J.; Engle, K. M. Directed, Nickel-Catalyzed Umpolung 1,2-Carboamination of Alkenyl Carbonyl Compounds. ACS Catal. 2019, 9, 224-229. (c) Liu, Z.; Wang, Y.; Wang, Z.; Zeng, T.; Liu, P, Engle, K. M. Catalytic Intermolecular Carboamination of Unactivated Alkenes via Directed Aminopalladation. J. Am. Chem. Soc. 2017, 139, 11261-11270.

(4) Selected examples: (a) Teng, S.; Jiao, Z.; Chi, Y. R.; Zhou, J. S. Asymmetric Wacker-Type Oxyallenylation and Azaallenylation of $\mathrm{Cy}$ clic Alkenes. Angew. Chem. Int. Ed. 2020, 59, 2246-2250. (b) Orcel, U.; Waser, J. Angew. Chem. Int. Ed. 2015, 54, 5250-5254. (c) Xie, Y.; $\mathrm{Hu}$, J.; Xie, P.; Qian, B.; Huang, H. Palladium-Catalyzed Difunctionalization of Enol Ehters to Amino Acetals with Aminals and Alcohols. J. Am. Chem. Soc. 2013, 135, 18327-18330. (d) Wang, Y.; Zhang, L.; Yang, Y.; Zhang, P.; Du, Z.; Wang, C. Alkene Oxyalkylation Enabled by Merging Rhenium Catalysis with Hypervalent Iodine(III) Reagents via Decarboxylation. J. Am. Chem. Soc. 2013, 135, 18048-18051. (e) Ball, L. T.; Lloyd-Jones, G. C.; Russel, C. A. Gold-Catalysed Oxyarylation of Styrenes and Mono- and gem-Disubstituted Olefins Facilitated by an Iodine(III) Oxidant. Chem. Eur. J. 2012, 18, 2931-2937. (f) Ball, L. T.; Green, M.; Lloyd-Jones, G. C.; Russel, C. A. Arylsilanes: Application to Gold-Catalyzed Oxyarylation of Alkenes. Org. Lett. 2010, 12, 4724-4727. (g) Brenzovich, W. E.; Brazeau, J.-F.; Toste, F. D. GoldCatalyzed Oxidative Coupling Reactions with Aryltrimethylsilanes. Org. Lett. 2010, 12, 4728-4731. (h) Melhado, A. D.; Brenzovich, W. E.; Lackner, A. D.; Toste, F. D. Gold-Catalyzed Three-Component Coupling: Oxidative Oxyarylation of Alkenes. J. Am. Chem. Soc. 2010, 132, 8885-8887. (i) Holton, R. A. Prostaglandin Synthesis via Carbopalladation. J. Am. Chem. Soc. 1977, 99, 8083-8085.

(5) (a) Hoffmann, R. W. Markovnikov Free Radical Addition Reactions, a Sleeping Beauty Kissed to Life. Chem. Soc. Rev. 2016, 45, 577583. (b) Tang, S.; Liu, K.; Liu, C.; Lei, A. Olefinic C - H Functionalization through Radical Alkenylation. Chem. Soc. Rev. 2015, 44, 10701082. (c) Tedder, J. M.; Walton, J. C. The Kinetics and Orientation of Free-Radical Addition to Olefins. Acc. Chem. Res. 1976, 9, 183-191.

(6) For a recent review, see: Jiang, H.; Studer, A. Intermolecular Radical Carboamination of Alkenes. Chem. Soc. Rev. 2020, 49, 1790-1811. Selected examples of three-component alkene carboamination reactions involving radical intermediates: (a) Xiong, Y.; Ma, X.; Zhang, G. Copper-Catalyzed Intermolecular Carboamination of Alkenes Induced by Visible Light. Org. Lett. 2019, 21, 1699-1703. (b) Jiang, H.; Seidler, G.; Studer, A. Carboamination of Unactivated Alkenes through ThreeComponent Radical Conjugate Addition. Angew. Chem. Int. Ed. 2019, 58, 16528-16532. (c) Xiao, H.; Shen, H.; Zhu, L.; Li, C. Copper-Catalyzed Radical Aminotrifluoromethylation of Alkenes. J. Am. Chem. Soc. 2019, 141, 11440-11445. (d) Zhang, Y.; Liu, H.; Tang, L.; Tang, H.-J.; Wang, L.; Zhu, C.; Feng, C. Intermolecular Carboamination of Unactivated Alkenes. J. Am. Chem. Soc. 2018, 140, 10695-10699. (e) Bao, X.; Yokoe, T.; Ha, T. M.; Wang, Q.; Zhu, J. Copper-Catalyzed Methylative Difunctionalization of Alkenes. Nat. Commun. 2018, 9, 3725. (f) Ouyang, X.-H.; Li, Y.; Song, R.-J.; Li, J.-H. Alkylamination of Styrenes with Alkyl $N$-Hydroxyphthalimide Esters and Amines by $\mathrm{B}\left(\mathrm{C}_{6} \mathrm{H}_{5}\right)_{3}$-Facilitated Photoredox Catalysis. Org. Lett. 2018, 20, 66596662. (g) Liu, Y.-Y.; Yang, X.-H.; Song, R.-J.; Luo, S.; Li, J.-H. Oxidative 1,2-Carboamination of Alkenes with Alkyl Nitriles and Amines Toward $\gamma$-Amino Alkyl Nitriles. Nat. Commun. 2017, 8, 14720. (h) Wang, D.; Wu, L.; Wang, F.; Wan, X.; Chen, P.; Lin, Z.; Liu, G. Asymmetric Copper-Catalyzed Intermolecular Aminoarylation of Styrenes: Efficient Access to Optical 2,2-Diarylethylamines. J. Am. Chem. Soc. 2017, 139, 6811-6814. (i) Wang, D.; Wang, F.; Chen, P.; Lin, Z.; Liu, G. Enantioselective Copper-Catalyzed Intermolecular Amino- and Azidocyanation of Alkenes in a Radical Process. Angew. Chem. Int. Ed. 
2017, 56, 2054-2058. (j) Qian, B.; Chen, S.; Wang, T.; Zhang, X.; Bao, H. Iron-Catalyzed Carboamination of Olefins: Synthesis of Amines and Disubstituted $\beta$-Amino Acids. J. Am. Chem. Soc. 2017, 139 , 13076-13082. (k) Zhang, W.; Pu, W.; Xiong, T.; Li, Y.; Zhou, X.; Sun, K.; Liu, Q.; Zhang, Q. Copper-Catalyzed Intermolecular Aminocyanation and Diamination of Alkenes. Angew. Chem. Int. Ed. 2013, 52, 2529-2533. Selected examples of three-component alkene carboetherification reactions involving radical intermediates: (1) Dong, Y.-X., Li, Y.; Gu, C.-C.; Jiang, S.-S.; Song, R.-J.; Li, J.-H. Copper-Catalyzed Three-Component Intermolecular Alkylesterification of Styrenes with Toluenes and Peroxyesters or Acids. Org. Lett. 2018, 20, 7594-7597. (m) Tlahuext-Aca, A.; Garza-Sanchez, R. A.; Glorius, F. Multicomponent Oxyalkylation of Styrenes Enabled by Hydrogen-Bond-Assisted Photoinduced Electron Transfer. Angew. Chem. Int. Ed. 2017, 56, 3708-3711. (n) Kindt, S.; Wicht, K.; Heinrich, M. R. Thermally Induced Carbohydroxylation of Styrenes with Aryldiazonium Salts. Angew. Chem. Int. Ed. 2016, 55, 8744-8747. (o) Wei, W.-T.; Li, H.-B.; Song, R.-J.; Li, J.-H. Copper-Catalyzed Oxidative Oxyalkylation of Enol Ethers with $\alpha$-Amino Carbonyl Compounds and Hydroperoxides. Chem. Commun. 2015, 51, 11325-11328. (p) Sazepin-Chatalova, C.; Wang, Q.; Sammis, G. M.; Zhu, J. Copper-Catalyzed Intermolecular Carboetherification of Unactivated Alkenes by Alkyl Nitriles and Alcohols. Angew. Chem. Int. Ed. 2015, 54, 5443-5446. (q) Liao, Z.; Yi, H.; Li, Z.; Fan, C.; Zhang, X.; Liu, J.; Deng, Z.; Lei, A. Copper-Catalyzed Radical Carbooxygenation: Alkylation and Alkoxylation of Styrenes. Chem. Asian J. 2015, 10, 96-99. (r) Yi, H.; Zhang, X.; Qin, C.; Liao, Z.; Liu, J.; Lei, A. Visible Light-Induced $\gamma$-Alkoxynitrile Synthesis via Three-Component Alkoxycyanomethylation of Alkenes. Adv. Synth. Catal. 2014, 356, 2873-2877. (s) Hari, D. P.; Hering, T.; Konig, B. The Photoredox-Catalyzed Meerwein Addition Reaction: Intermolecular Amino-Arylation of Alkenes. Angew. Chem. Int. Ed. 2014, 53, 725-728. (t) Fumagalli, G.; Boyd, S.; Greaney, M. F. Oxyarylation and Aminoarylation of Styrenes Using Photoredox Catalysis. Org. Lett. 2013, 15, 4398-4401.

(7) (a) Gockel, S. N.; Buchanan, T. L.; Hull, K. L. Cu-Catalyzed ThreeComponent Carboamination of Alkenes. J. Am. Chem. Soc. 2018, 140, 58-61. (b) Pan, G.-H.; Song, R.-J.; Xie, Y.-X.; Luo, S.; Li, J.-H. Synthesis of $\gamma$-Amino Esters by Copper-Catalyzed Intermolecular 1,2Aminoalkylation of Alkenes with Amines and $\alpha$-Bromoalkyl Esters. Synthesis 2018, 50, 1651-1660. (c) Pan, G.-H.; Ouyang, X.-H.; Hu, M.; Xie, Y.-X.; Li, J.-H. Copper-Catalyzed Intermolecular Aminoalkylation of Alkenes with $\alpha$-Bromoalkyl Esters and Amines toward Pyrrolidin-2-ones. Adv. Synth. Catal. 2017, 359, 2564-2570.

(8) Caruano, J.; Muccioli, G. G.; Robiette, R. Biologically Active $\gamma$ Lactams: Synthesis and Natural Sources. Org. Biomol. Chem. 2016, 14, 10134-10156.

(9) (a) Lv, Y.; Pu, W.; Mao, S.; Ren, X.; Wu, Y.; Cui, H. Cu-Catalyzed Intermolecular Oxyalkylation of Styrenes Under Air: Access to Diverse Iminolactones. $R S C A d v$. 2017, 7, 41723-41726. (b) Nishikata, T.; Itonaga, K.; Yamaguchi, N.; Sumimoto, M. Amine-Controlled Divergent Reaction: Iminolactonization and Olefination in the Presence of a Cu(II) Catalyst. Org. Lett. 2017, 19, 2686-2689.

(10) Pan, G.-H.; Song, R.-J.; Li, J.-H. Radical-Mediated Synthesis of $\gamma$-Lactones by Copper-Catalyzed Intermolecular Carboesterification of Alkenes with $\alpha$-Carbonyl Alkyl Bromides and $\mathrm{H}_{2} \mathrm{O}$. Org. Chem. Front. 2018, 5, 179-183

(11) (a) Lanke, V.; Marek, I. Stereospecific Nucleophilic Substitution at Tertiary and Quaternary Stereocenters. Chem. Sci. 2020, 11, 93789385. (b) Pronin, S. V.; Reiher, C. A.; Shenvi, R. A. Stereoinversion of Tertiary Alcohols to Tertiary-Alkyl Isonitriles and Amines. Nature 2013, 501, 195-300.

(12) (a) Krapcho, A. P.; Glynn, G. A.; Grenon, B. J. Tetrahedron Lett. 1967, 8, 215-217. (b) Lee, A.; Michrowska, A.; Sulzer-Mosse, S.; List, B. Angew. Chem., Int. Ed. 2011, 50, 1707-1710.
(13) Chakraborti, A. K.; Nayak, M. K.; Sharma, L. Selective Deprotection of Aryl Acetates, Benzoates, Pivalates, and Tosylates under Nonhydrolytic and Virtually Neutral Conditions. J. Org. Chem. 1999, 64, 8027-8030. 